\title{
EL MARKETING DIGITAL Y SU INFLUENCIA EN LA CAPTACIÓN DE CLIENTES EN LAS PYMES DE ECUADOR
}

\author{
Ing. Deodato Loor Chávez PhD. \\ Universidad Laica Eloy Alfaro de Manabí \\ ORCID: https://orcid.org/0000-0001-8123-4543 \\ deodato.loor@uleam.edu.ec \\ Ing. Cristhian López Leones Mg. \\ Universidad Laica Eloy Alfaro de Manabí \\ ORCID: https://orcid.org/0000-0002-2963-1135 \\ cristhian.lopez@uleam.edu.ec \\ Ing. Jimmy Molina Cedeño Mg. \\ Universidad Laica Eloy Alfaro de Manabí \\ ORCID: https://orcid.org/0000-0002-3004-0893 \\ ¡immy.molina@uleam.edu.ec
}

Para citar este artículo puede utilizar el siguiente formato:

Deodato Loor Chávez, Cristhian López Leones y Jimmy Molina Cedeño: "El marketing digital y su influencia en la captación de clientes en las PYMES de Ecuador", Revista Caribeña de Ciencias Sociales (vol 10, № 8 octubre-diciembre 2021, pp. 37-48. En línea:

https://doi.org/10.51896/caribe/GEUL6587

\section{RESUMEN}

Definido también como «Marketing Online» (MO), la «Mercadotecnia Digital» (MD) y su implementación a nivel empresarial, potencializa el uso de las redes sociales, no solo a nivel de unidades de negocio, sino de consumidores, creando un proceso de interacción comercial de doble vía entre empresa-cliente, lo cual sin duda ayuda atraer consumidores, con un consecuente incremento en las ventas de bienes y servicios. En el Ecuador, a raíz de la pandemia del Covid-19, la utilización de canales de comunicación masiva, han sido el mejor aliado para todo tipo y tamaño de compañía, transformándose el «MD» en el elemento diferenciador en muchas organizaciones, toda vez que no solo se aprovechan los medios de alta audiencia, sino que se implementan estrategias diferenciadas para la llegar a los compradores. El objetivo planteado en esta investigación fue "establecer de que forma el Marketing Digital influye en la captación de clientes en las PYMES de Ecuador". Bajo la aplicación del método inductivo completo, empleando varios tipos de investigación, se abordan muchos problemas que ralentizan los procesos en las pequeñas y medianas empresas, dificultándoles efectuar el salto estratégico y tecnológico para mejorar sus operaciones comerciales. Así mismo se formularon hipótesis que permitan medir las correlaciones entre las variables elegidas, sus dimensiones e indicadores. Luego de emplear el sistema estadístico SPSS-25, "Ios coeficientes de Alfa de Cronbach y $p$ Rho de Spearman", se obtienen 
importantes resultados que develan cuan ligadas se encuentran las PYMES a las nuevas tendencias de negocios digitales.

Palabras clave: Desarrollo tecnológico, Comercialización, Captación de clientes, Marketing, Medios digitales.

\title{
DIGITAL MARKETING AND ITS INFLUENCE ON CUSTOMER ATTRACTING IN ECUADOR SMES
}

\begin{abstract}
:
Also defined as "Online Marketing» (MO), «Digital Marketing» (MD) and its implementation at the business level, potentiates the use of social networks, not only at the level of business units, but also of consumers, creating a two-way commercial interaction process between company-client, which undoubtedly helps attract consumers, with a consequent increase in sales of goods and services. In Ecuador, as a result of the Covid-19 pandemic, the use of mass communication channels has been the best ally for all types and sizes of company, transforming the «MD» into the differentiating element in many organizations, every time that not only high-audience media are used, but differentiated strategies are implemented to reach buyers. The objective set out in this research was "to establish how Digital Marketing influences customer acquisition in SMEs in Ecuador". Under the application of the complete inductive method, using various types of research, many problems are addressed that slow down processes in small and medium-sized companies, making it difficult for them to make the strategic and technological leap to improve their business operations. Likewise, hypotheses were formulated that allow measuring the correlations between the chosen variables, their dimensions and indicators. After using the SPSS-25 statistical system, "Cronbach's Alpha coefficients and Spearman's $p$ Rho", important results are obtained that reveal how linked SMEs are to new digital business trends.
\end{abstract}

Keywords: Technological development, Marketing, Customer acquisition, Marketing, Digital media.

\section{INTRODUCCIÓN}

Con más de dos décadas de vigencia y una fuerte influencia en los negocios globales, el Marketing Digital (MD), le ha permitido a las empresas mejorar sus relaciones con los clientes, posicionar sus marcas, incrementar su nivel de facturación, con una consecuente participación de mercado. De esta tipología de mercadeo, nacen otras líneas que se fusionan para una adecuada estrategia de «MD» como el «Social Media Marketing y la Mercadotecnia en Redes Sociales», todas empujadas por el desarrollo tecnológico, como principal instrumento y vía de contacto entre consumidores y comparadores. Multinacionales como Amazon entendieron el concepto de esta forma de hacer negocios, sin duda tan exitosas como para otorgarle a muchas, la impronta de líderes, como es el caso de JD, "Alibaba, eBay», la misma Amazon que lidera el top ten, entre muchas más. 
De a poco la promoción en medios clásicos como la prensa, radio, televisión (por sus elevados costos), ha sido desplazada por el internet, con un mercado potencial conectado en red, principalmente aquellos grupos etarios en edades comprendidas entre 17 a 55 años, muy dados al manejo de dispositivos como los Smartphone, Tablets, IPhone, entre otros, de diferentes productores. Estas son las herramientas que están aprovechando las organizaciones para repuntar su comercialización, más en estos tiempos de confinamiento y distanciamiento social, causado por la epidemia global del SARS-COV-2. El «MD» desdobla potentes acciones, cuando a través de un plan combinado de marketing y sus distintos tipos, se plantean estrategias y tácticas apropiadas para el target objetivo, entre ellas el E-mail Marketing, de contenidos, de buscadores, en redes sociales, de afiliación, de posicionamiento en buscadores, de influencers, publicidad en redes, entre otras. La vertiginosa utilización de medios digitales, calculada en promedio de 6.7 horas al día por persona en edad adulta (HubSpot, 2020, p.10), es una gran oportunidad para que las micro, pequeñas, medianas y grandes empresas, generen su espacio de crecimiento, en tanto y en cuanto se apropien de las Tics. El contexto de análisis efectuado en la delimitación espacial del objeto de estudio (PYMES de Ecuador), tiene su propio escenario; por un lado coexisten los problemas típicos del tamaño de las unidades de negocio, como la limitada infraestructura física, tecnológica, recurso humano desprovisto de capacitación, falta de liquidez para invertir en procesos operativos, comerciales y de mercadeo, hacen parte de las carencias en muchas organizaciones, sin importar su envergadura, dificultades que se han agravado en este contexto critico de pandemia. Por ello sobreviene la pregunta problemica: ¿de que forma el Marketing Digital influye en la Captación de Clientes en las PYMES de Ecuador?, misma que se relaciona directamente con el objetivo descrito en el resumen.

\section{Que deben integrar las empresas del Ecuador para una buena gestión de «MD»}

Sin distinción de tamaño, toda compañía debe desarrollar un «plan estratégico integrado», el cual sirva como una hoja de ruta para trazar sus metas, las mismas que se podrán lograr con tácticas adecuadas en toda área, pero sin duda más en la comercial, que es donde afianza el marketing digital y su aporte para maximizar los esfuerzos organizacionales, con estrategias tales como:

- Generación de un contenido altamente llamativo para el público online.

- Creatividad en el diseño de las páginas web, con calidad de información.

- Tener un buen conocimiento del comportamiento de los clientes regulares y potenciales.

- Aprovechar las distintas redes de comunicación social.

- Participar e interactuar con los consumidores para crear una comunidad activa entre empresacliente.

- Tener personal capacitado, principalmente un profesional preparado que maneje la parte del desarrollo tecnológico en su conjunto, lo que en las grandes transnacionales sería un ejecutivo CEO. 
- De los puntos anteriores se desprende la gestión para posicionar la marca/empresa/producto en los principales motores de búsqueda, para captar nuevos clientes.

\section{El valor añadido para las empresas empleando el «MD»}

Las empresas tienen oportunidades en igualdad de condiciones, solo se van a diferenciar probablemente por la distinción de sus marcas, lo cual si genera sin dudas una mayor notoriedad e impacto, mientras se aprovechen con buenas estrategias estos elementos, y, para ello es vital que los gestores de marketing digital (CEO), estén a la altura de lo requerido por el mercado cibernauta. Otros aspectos de valor añadido del «MD» son por ejemplo la penetración geográfica, la medición resultados de forma más práctica e inmediata ( $\mathrm{ROI}$ de acciones), la personalización de relaciones con los clientes, abaratando el costo que se generaría en una visita personal, típica en el marketing tradicional; así mismo, es posible prospectar, captar y fidelizar consumidores de manera más rápida.

Otro beneficio es que se puede clasificar y segmentar la audiencia en función de edades, género, estatus socioeconómico, grado educativo, sitio de residencia, aficiones, estilo de vida e incluso efectuar una aproximación de la propia personalidad del individuo, entre otros aspectos agregados para mejorar los resultados comerciales. En todo caso, la mercadotecnia digital y sus acciones, son enteramente moldeables, es decir permiten trabajar sobre la marcha en el direccionamiento de aquellos resultados que por alguna acción inadecuada no son satisfactorios para la empresa, además de tener en cuenta que la implementación de una campaña digital es mucho más reducida presupuestariamente, en relación a la del marketing clásico, que suele ser mas de campo y por ende costosa.

\section{Principales redes sociales}

Tras el avance de este artículo, queda bastante claro que uno de los fines principales del «MD» es aprovechar las redes sociales para ampliar la notoriedad de las organizaciones y sus marcas, cuyo contenido promocional debe ser lo suficientemente cautivador para captar la atención del cliente, y entregar un mejor servicio, por aquello la selección de los canales digitales cobra relevancia para desarrollar comunidades de consumidores y convertirlos también medios directos para forjar ventas. De esta forma, las redes sociales más apropiadas para la interrelacionarse con los clientes son: «Facebook, Twitter, Instagram, Linkedln, YouTube, Snapchat, TikTok y WhatsApp». Complementariamente es importante que las empresas creen sus propios blogs y páginas web, como soporte de apoyo comunicacional.

\section{Fundamentación teórica de las variables Marketing Digital}

La concepción más clara y llana del marketing digital es aquella que se identifica con el «marketing online o en línea», que no es otra cosa que aprovechar las «redes sociales y el internet» para impulsar 
electrónicamente el comercio de tangibles e intangibles. Kotler y Armstrong (2013), definen al «MD» como "un mecanismo para que una empresa trate de darse a conocer, promover y vender productos o servicios por medio de internet, abarcando nuevos nichos de mercado, abasteciendo las necesidades del consumidor" (p.149).

En ese mismo contexto Kevin Green (2020) de la multinacional Dell, acopia la definición de ver al "Marketing Digital como la punta de lanza para interactuar con la audiencia o personas deseadas; no es un simple canal de transmisión, sino la manera en que se hace cobrar vida a una experiencia por medio de los canales" (p. 1). De otro lado Rachael Marret (2018) de Campbell Mithun, manifiesta que "el marketing a través de canales va más allá de la mensajería, puesto que las marcas pueden hacer conexión con los consumidores en línea, por medio de entornos virtuales, app móviles, medios de pagos, blogs, entre otros". (p.1)

\section{Captación de clientes}

Captar clientes es una de las tareas más indispensables en una organización, pues el sostenimiento de la misma depende en gran medida del poder atraer más consumidores, para ello el gran desafío que tiene el mercadologo es implementar las estrategias más idóneas para lograr este propósito, que tiene que ver en paralelo con la calidad de atención y servicio al cliente, como también con una adecuada segmentación de mercado para conocer más de cerca las necesidades de un target definido. Las empresas que se insertan en este propósito cotidiano, mejorar ostensiblemente su facturación, así como de manera automática su participación de mercado y posicionamiento de marca, con una alta posibilidad de expansión. La «Mercadotecnia Digital» es uno de los instrumentos más competentes para prospectar/captar/retener o fidelizar clientes, pues como medio generador de estrategias de contacto con los consumidores, posibilita la perdurabilidad de la relación, empleando por ejemplo el multicanal para ganar presencia en los distintos canales donde se encuentra el cliente objetivo; así mismo el Inbound Marketing, cuyo enfoque es lograr atraer más clientes potenciando contenidos, el social media y el SEO (buscadores).

Siempre es importante teorizar científicamente lo que se quiere articular, así por ejemplo Regalado et al., (2011), sobre la captación de clientes señalan que "consiste en desarrollar un proceso clave para establecer relaciones firmes y duraderas con las personas o las organizaciones que, directa 0 indirectamente pueden influir en el éxito de las actividades de la empresa" (p.16). Mesen, J. (2011) mientras tanto considera sobre el mismo concepto que, "concierne a la atracción, desarrollo, mantenimiento y retención de relaciones con los clientes. La creación de clientes leales es el eje central de la captación, para conseguirlo, las empresas deben acrecentar los lazos con sus clientes" (p.31). Los autores modernos consideran que la tecnología revoluciono la manera de hacer negocios, lo cual significa que las organizaciones deben preparar cada día sus áreas comerciales y de marketing, 
desarrollando en el orden estratégico y operativo, acciones que admitan lograr el gran objetivo de captar clientes. Por ello autores como Kotler et al (2000) determinan que "el marketing se está convirtiendo en una batalla más en la información, que en el poder de las ventas" (p.38).

\section{Hipótesis de la investigación}

Tabla 1

Formulación de hipótesis

\begin{tabular}{ll}
\hline \multicolumn{1}{c}{ Hipótesis } & \multicolumn{1}{c}{ Formulaciones } \\
\hline General & $\begin{array}{l}\text { X. El marketing digital influye en la captación de clientes en las PYMES de Ecuador } \\
\text { X1. La implementación de herramientas tecnológicas influye en la captación de clientes }\end{array}$ \\
Especifica 1. & $\begin{array}{l}\text { en las PYMES de Ecuador. } \\
\text { X2. La funcionalidad del sitio web influye en la captación de clientes en las PYMES de } \\
\text { Especifica 2. }\end{array}$ \\
Espuador. & $\begin{array}{l}\text { X3. La capacitación del recurso humano influye en la captación de clientes en las } \\
\text { PYMES de Ecuador. }\end{array}$ \\
\hline
\end{tabular}

Fuente: Autores (2021)

\section{METODOLOGÍA}

Para el presente estudio se aplica el "método inductivo completo", pasando de aspectos generales a particulares. De igual manera se emplea la investigación "documental, exploratoria, descriptiva, explicativa, de campo, cualitativa, cuantitativa y correlacional". Por medio del estadístico SPSS-25 se establecen las correlaciones entres las variables y dimensiones, permitiendo dicho programa validar las hipótesis, como la fiabilidad del instrumento (encuesta), como herramienta del estudio cualitativo, que permitió conjuntamente con técnicas como la observación y entrevista, recabar una importante base de elementos e información para enriquecer la recolección de datos, que apuntan a la comunicación con el encuestado y/o entrevistado, y descubrir lo que piensan y porque. Luego viene la conversión de datos de tipo cuantitativo.

\section{Diseño}

Se diseñó un instrumento "policotomico" tipo escala de Likert, el cual se aplicó a directores/gerentes/administradores/jefes de área de las PYMES del Ecuador, tomando una muestra por las provincias y ciudades más trascendentales del país estudiado. El contenido de la encuesta abarca principalmente los problemas que envuelven a las empresas, en torno a la aplicación del Marketing Digital, para conocer sus fortalezas y debilidades en cuanto a su implementación, y como ha servido para la captación de clientes. El criterio de selección del grupo de estudio, se fundamenta en lo señalado por Hernández, Fernández y Batista (2014), quienes en cita textual indican: "se consiente receptar la información en un período determinado, para conseguir datos relevantes sobre el grupo poblacional objetivo" (p.40). 


\section{Unidad de análisis y población de estudio}

Son parte de la unidad de análisis las "PYMES de Ecuador", en función del Directorio de Empresas del Instituto Nacional de Estadísticas y Censos (INEC, 2019). De acuerdo a la tabla 2, se evidencia la distribución de las pequeñas y medianas empresas en la nación objeto de estudio, con una población de 76.101 unidades de negocio.

\section{Tabla 2}

PYMES registradas en Ecuador

\begin{tabular}{lc}
\hline Tamaño de empresa & No. Empresas \\
\hline Pequeña empresa & 61.759 \\
Mediana empresa "A" & 8.544 \\
Mediana empresa "B" & 5.798 \\
Total & $\mathbf{7 6 . 1 0 1}$ \\
\hline
\end{tabular}

Fuente: INEC (2019)

\section{Muestra}

La extrapolación de la muestra se da a través de un muestreo aleatorio simple, con sustento teórico en los fundamentos de (Kish, 1995; Kalton y Heeringa, 2003, p.57) citados por Hernández et al., (2014), quienes indican que "se insta en la necesidad de conocer el tamaño del error y el nivel de confianza" (p.72). Así mismo para la determinación de la muestra, se aplica la formula finita, con el siguiente procedimiento y sus resultados:

$\mathrm{n}=\frac{\mathrm{Z}^{2} \cdot \mathrm{P} \cdot \mathrm{Q} \cdot \mathrm{N}}{\mathrm{Z}^{2} \cdot \mathrm{P} \cdot \mathrm{Q}+(\mathrm{N})(\mathrm{e})^{2}}$

\section{Dónde:}

$\mathrm{N}=$ Total de la población universo (76.101)

$\mathrm{n}=$ Tamaño de la muestra (?)

$Z$ = Nivel de confianza (1.96)

$\mathrm{P}=$ Probabilidad de aceptación (0.5)

$\mathrm{Q}=$ Probabilidad de rechazo $(0.5)$

$\mathrm{e}=$ Margen de error de muestreo (0.05)

$\mathrm{n}=\frac{1,96^{2} *(0,5) *(0,5) *(76.101)}{1.96^{2} *(0.5) *(0.5)+(76.101) *(0,05)^{2}}$

$\mathrm{n}=\frac{3,8416 * 0,25 * 76.101}{3,8416 * 0,25+76.101 * 0.0025}$

$$
\mathrm{n}=\frac{73087,40}{191_{s} 21}=382
$$

Aplicada la formula y despejados los datos, se estableció una muestra de 382 encuestas, las cuales se corrieron en cinco importantes provincias del Ecuador, detalladas en la tabla 3 : 


\section{Tabla 3}

Muestra tomada por provincias de Ecuador.

\begin{tabular}{|c|c|c|c|c|c|c|c|}
\hline \multicolumn{8}{|c|}{ Muestra por PYMES (Provincias - Ecuador) } \\
\hline $\begin{array}{c}\text { Tamaño } \\
\text { de } \\
\text { empresa }\end{array}$ & $\begin{array}{c}\# \\
\text { Empresas }\end{array}$ & Pichincha & Guayas & $\begin{array}{l}\text { Muestra } \\
\text { Manabí }\end{array}$ & Azuay & El Oro & $\begin{array}{c}\text { Total } \\
\text { Muestra }\end{array}$ \\
\hline $\begin{array}{l}\text { Pequeñas } \\
\text { empresas }\end{array}$ & 61.759 & 85 & 55 & 30 & 25 & 20 & 215 \\
\hline $\begin{array}{c}\text { Medianas } \\
\text { empresas } \\
\text { A }\end{array}$ & 8.544 & 30 & 25 & 15 & 15 & 10 & 95 \\
\hline $\begin{array}{c}\text { Medianas } \\
\text { empresas } \\
\text { B }\end{array}$ & 5.798 & 25 & 23 & 8 & 9 & 7 & 72 \\
\hline Totales & 76.101 & 140 & 103 & 53 & 49 & 37 & 382 \\
\hline
\end{tabular}

Fuente: INEC (2019) - Adaptación: Autores

\section{Datos recolectados}

Se recaba la información por medio del instrumento (encuesta en escala de Likert), la cual cuenta con una distribución de 30 preguntas, (15 por variable). En función de la escala normativa, cada ítem tiene asignado una valoración, consintiendo medir la jerarquía de respuesta; así por ejemplo: nunca equivale a (1), raramente (2), ocasionalmente (3), frecuentemente (4) muy frecuentemente (5).

\section{RESULTADOS}

Aplicando el coeficiente "Alfa de Cronbach por medio del SPSS-25 se validó el "instrumento", reflejando un nivel de confiabilidad de 0,992, deduciéndose un "nivel de correlación muy alto". (Tabla 4).

Tabla 4

Alfa de Cronbach

\begin{tabular}{rr}
\hline \multicolumn{2}{c}{ Estadísticas de fiabilidad } \\
\hline Alfa de Cronbach & $\mathrm{N}$ de elementos \\
, 992 & 30 \\
\hline
\end{tabular}

Recurso: Estadístico SPSS-25

\section{Hipótesis (pruebas y validación)}

La validación de las hipótesis se dio por medio del recurso estadístico SPSS-25, empleando el coeficiente correlacional "Rho de Spearman", bajo un trazo longitudinal entre variables y dimensiones. 


\section{Tabla 5}

Resumen de validación de hipótesis

\begin{tabular}{cccccc}
\hline Hipótesis & $\begin{array}{c}\text { Variable } \\
\text { Independiente }\end{array}$ & $\begin{array}{c}\text { Variable } \\
\text { Dependiente }\end{array}$ & Sig & $\begin{array}{c}\text { Rho de } \\
\text { Spearman }\end{array}$ & Resultado \\
\hline H. General & X. Marketing digital &, 000 &, 850 & Se rechaza Ho \\
H. Específica & $\begin{array}{c}\text { X1. Herramientas } \\
\text { tecnológicas }\end{array}$ & $\begin{array}{c}\text { Y. Captación } \\
\text { de clientes }\end{array}$ &, 000 &, 890 & Se rechaza Ho \\
H. Específica & $\begin{array}{c}\text { X2. Funcionalidad } \\
\mathbf{2}\end{array}$ & $\begin{array}{c}\text { del sitio Web } \\
\text { H. Específica } \\
\mathbf{3}\end{array}$ & $\begin{array}{c}\text { X3. Capacitación del } \\
\text { recurso humano }\end{array}$ &, 000 & Se rechaza Ho \\
** La correlación es significativa en el nivel de 0,01 (bilateral) & &, 960 & Se rechaza Ho \\
\hline
\end{tabular}

Recurso: Estadístico SPSS-25

Tabla 6

Interpretaciones

\section{Hipótesis General (X)}

H1. El marketing digital SI influye significativamente en la captación de clientes en las PYMES de Ecuador.

\section{Análisis}

La prueba de la hipótesis general (X), en función de los resultados del SPSS-25, despliegan una significación bilateral de 0,000 y un coeficiente de correlación p "Rho" de Spearman de 0,850, simbolizando la existencia de una "correlación muy buena". En consecuencia, realizado el cruce entre las variables (independiente y dependiente), se elimina la hipótesis nula $(\mathrm{H} 0)$, y se acepta la alternativa $(\mathrm{H} 1)$.

\section{Hipótesis especifica X1}

H1. Las herramientas digitales SI influyen significativamente en la captación de clientes en las PYMES de Ecuador.

\section{Análisis}

Para la hipótesis especifica (X1), se obtiene una significación bilateral de 0.000 , y un coeficiente $p$ (Rho) Spearman de 0,890, eliminando la hipótesis nula $(\mathrm{H} 0)$, admitiendo la alternativa $(\mathrm{H} 1)$, dada la existencia de una "correlación muy buena".

\section{Hipótesis especifica X2}

H2. La funcionalidad del sitio Web SI influye significativamente en la captación de clientes en las PYMES de Ecuador.

\section{Análisis}


De acuerdo a los resultados alcanzados en la hipótesis especifica (X2), existe una significación bilateral de 0.000 , con un coeficiente $p$ (Rho) Spearman de 0,880 , descartando la hipótesis nula $(\mathrm{H} 0)$, afirmando la hipótesis alternativa $(\mathrm{H} 1)$, con una "correlación muy buena".

\section{Hipótesis especifica X3}

H3. La capacitación del recurso humano SI influye significativamente en la captación de clientes en las PYMES de Ecuador.

\section{Análisis}

En la hipótesis especifica (X3), el programa estadístico presenta una significación bilateral de 0.000 , y un coeficiente $p$ (Rho) Spearman de 0,960, excluyendo la hipótesis nula (H0), aceptando la alternativa (H1), por lo que se interpreta como una "correlación muy buena".

Fuente: Autores (2021)

\section{DISCUSIÓN}

Los medios digitales se han transformado en una herramienta vital para la convivencia humana, dado que facilitan de manera efectiva la comunicación entre sujeto/familia/entorno/sociedad/empresa, volviéndose tan necesaria y funcional en estos tiempos de interacción social. El aprovechamiento de los canales tecnológicos, le permite al hombre moderno estudiar, aprender, comprar, negociar, promocionar, etc., en esta considerada la tercera revolución industrial, volcada hacia la tecnología. Las empresas se han inteligenciado para integrar este recurso y desdoblar su capacidad productiva, pero sobre todo comercial, incorporando la "gestión del marketing digital" como el elemento que agrega valor a la posición de la organización en el mercado. Esta nueva forma de operar, les va a permitir a las empresas volverse competitivas, principalmente a las PYMES, y sobre todo a las de Ecuador. El estudio devela que las empresas que se han innovado tecnológicamente, tendrán más posibilidad de sostenerse en el tiempo, explotando al máximo las bondades que provee la "mercadotecnia digital", acogiendo en paráfrasis la cita famosa del magnate de la tecnología Bill Gates, quien señala "que si un negocio no tiene presencia en internet, no existe".

\section{CONCLUSIONES}

1. La investigación cumplió finalmente con el objetivo trazado, cual fue "establecer de que forma el Marketing Digital influye en la captación de clientes en las PYMES de Ecuador", con resultados técnicos/estadísticos que validaron el cumplimento de esta meta, tras levantar importante información en las empresas objeto de estudio a nivel de Ecuador.

\section{En función de las hipótesis:}


2. Respecto a la hipótesis general $(\mathrm{X})$, se demostró la existencia de una "correlación muy buena" con un $\mathrm{p}$ "Rho de Spearman" de 0,850, concluyéndose que: "las PYMES de Ecuador que han aplicado el marketing digital, han logrado mayores resultados en la captación de clientes".

3. Se prueba a través de la hipótesis especifica (X1), que existe una "correlación muy buena" con un $\mathrm{p}$ "Rho de Spearman" de 0,890, destacándose que "la implementación de herramientas tecnológicas, agregan potencialidad a gestión operacional de las PYMES de Ecuador, contribuyendo a la captación de clientes".

4. En lo que concierne a la hipótesis especifica (X2), se determina una "correlación muy buena", permitiendo concluir que: "la funcionalidad del sitio web optimiza la gestión comercial de las PYMES, favoreciendo a la captación de clientes".

5. Finalmente, en la prueba de hipótesis especifica (X3), se logró evidenciar una "correlación muy buena", denotándose haber alcanzado una "correlación muy buena", cuya interpretación permite señalar que: "la capacitación del recurso humano es vital en el propósito de la captación de clientes en las PYMES de Ecuador", luego de haber comparado resultados en empresas que si apuestan a su talento humano.

\section{REFERENCIAS}

Hernández, R., Fernández, C. y Batista, P. (2014). Metodología de la Investigación. Ed. MacGraw-Hill. México.

HubSpot (2020). Estadísticas de Marketing. Nombre de sitio url: https://www.hubspot.es/marketingstatistics

Instituto Nacional de Estadísticas y Censos del Ecuador (2019). Boletín anual. Ed. INEC. Ecuador.

Kalton, G; Heeringa, S.; (2003). Artículos seleccionados en metodología de la encuesta. Ed. WileyIntersciene. ISBN. 978-0471266612.

Kevin Green (2010). Mercadeo digital. Nombre del sitio url: https://joseluispg.com/marketing-digitaldefinicion-de-autores/.

Kish, L. (1995). Diseño estadístico para la investigación. Ed. CIS. España.

Kotler, Philip y Armstrong, Gary (2013). Fundamentos de Marketing. Ed. Pearson. México.

Kotler, P.; Cámara, D.; Grande, I.; Cruz, I. (2000). Dirección de Marketing, Edición del Milenio. Ed. Prentice Hall Iberia. España.

Mesen, J. (2011). Mercadotecnia. Ed. Limusa. México.

Rachael Marret (2018). Marketing Digital. Nombre del sitio url: https://joseluispg.com/marketing-digitaldefinicion-de-autores/. 
Regalado, O., Allpaca, R., Baca, L., Gerónimo, M. (2011). Endomarketing: estrategias de relación con el cliente interno. Ed. ESAN. Perú. 